\title{
Do They Even Care? Measuring Instructor Value of Student Privacy in the Context of Learning Analytics
}

\author{
Kyle M. L. Jones \\ Indiana University- \\ Indianapolis (IUPUI) \\ kmlj@iupui.edu
}

\author{
Amy VanScoy \\ University at Buffalo \\ vanscoy@buffalo.edu
}

\author{
Kawanna Bright \\ East Carolina University \\ brightka19@ecu.edu
}

\author{
Alison Harding \\ University at Buffalo \\ ahall5@buffalo.edu
}

\begin{abstract}
Learning analytics tools are becoming commonplace in educational technologies, but extant student privacy issues remain largely unresolved. It is unknown whether or not faculty care about student privacy and see privacy as valuable for learning. The research herein addresses findings from a survey of over 500 full-time higher education instructors. The findings detail faculty perspectives of their own privacy, students' privacy, and the high degree to which they value both. Data indicate that faculty believe that privacy is important to intellectual behaviors and learning. This work reports initial findings of a multi-phase, grant-funded research project that will further uncover instructor views of learning analytics and its student privacy issues.
\end{abstract}

\section{Introduction}

Higher education institutions are increasing their capacity for and capability with learning analytics technologies. While research is still needed to see if this sociotechnical practice will produce notable benefits, institutions continue to push forward. Researchers and pundits alike point out that learning analytics raises significant student privacy issues and other ethical concerns. While students may be the primary subjects of learning analytics, it is fair to characterize faculty as the primary users, as they are the ones that utilize data and dashboards to effect instructional change and intervene with students. However, faculty views of learning analytics are under researched, especially regarding student privacy. This paper reports finding from a national survey of over 500 full-time higher education instructors to begin to fill this identified gap.

The paper begins with a review of the literature framed by a tight summary of learning analytics and a more expansive discussion of the privacy and ethics issues. The methods section follows, which includes a description of our survey's design and distribution strategy. In the findings, we home in on key data indicating how faculty define student privacy and the high degree to which faculty value their students' privacy. Our discussion raises questions about the homogeneity of the data and opportunities for further inquiry.

\section{Literature review}

\subsection{Learning analytics}

The interest in aggregating, mining, and analyzing troves of student data continues. Often under the auspices of "learning analytics" and "educational data mining," these sociotechnical practices attempt to describe, predict, and intervene at sites of learning to improve learning outcomes [1], [2]. They also strive to mold the holistic student experience in such a way that student success-which is to say measures of academic achievement, retention, and graduationincreases, usually while reducing institutional labor and resource expenditures. The data infrastructures on which learning analytics rely serve as new kinds of "policy instruments" that assist administrators to structure academic labor and intellectual behavior for faculty and students alike [3], [4].

On the face of it, these goals are worthy endeavors. Where American higher education is concerned, the cost of pursuing an undergraduate or graduate degree can be, and often is, a challenging financial ask. Failure to earn one's degree can lead to years of economic hardship as individuals pursue financially beneficial endeavors while shouldering significant debt [5]. Additionally, universities compete in a highly competitive market to attract students and demonstrate institutional superiority via rankings systems. If institutions can adopt data science 
strategies that help students achieve success (and reduce the risk of socioeconomic ruin) while improving their institutional reputation in the process, then data mining and predictive analytics seem justified (see [6]). But do the ends justify the means?

More often than not, learning analytics advocates predicate their work on the repeated Big Data principle that more data is better data [7], [8]. This essential position has motivated institutional professionals and researchers alike to push the "data frontier" [9, p. 18] even further by scanning for revelatory data sources that make visible student lives for analysis and intervention. Citing Solove [10] and Jones [11], respectively, Kwet and Prinsloo [12, p. 511] note that "higher education institutions are extending the data frontier through 'smart campus' and 'smart classroom'[projects].... As such, physical space, and the broader project of student data collection, is becoming part of an 'elaborate lattice of information networking' where data are shared, sold, merged, and new data bodies and data profiles come into existence." Quite obviously, learning analytics has a narrow privacy problem and a broader, more complex set of ethical quandaries.

\subsection{Privacy and ethics issues}

Since its inception, learning analytics has struggled with significant privacy and ethics issues, for there are no easy answers to these very real questions. Some practitioners have gone as far to suggest that the ethical responsibility is overly burdensome [13]. They argue that the breadth and depth of moral questions learning analytics raises has paralyzed the implementation and growth of learning analytics. We will not attempt to comprehensively catalog the privacy and ethics issues (see [14]-[18], [19]). However, we will contextualize these things in relation to our primary concern: instruction and learning.

Scholars have identified student privacy as a critical condition necessary to enable and protect the pursuit of ideas and development speech [20]. Students may alter their natural behaviors when digitally surveilled by reducing their engagement in contentious but important debates around politics, religion, and social values. To this point, HartmanCaverly [21, p. 39] states that "leaderboards and other forms of social surveillance in analytics-enhanced courseware engender student performativity that exerts a chilling effect on learning behavior, resulting in self-censorship, inhibition, silencing, and conformity." Polonetsky and Tene [22, p. 982] write with stark language that for students "who believe that every page view and quiz response will be recorded and analyzed, life under the magnifying glass becomes a grueling and relentless test." Some degree of surveillance is a basic requisite for learning analytics in order to support interventions, which raise other concerns.

Learning analytics emerged in tandem with the growing use of data dashboards in support of business intelligence purposes. Many scholars posit that these dashboards can equally help faculty and students. For faculty, they can make obvious students who are at academic risk using descriptive data and predictive measures [23]. For students, dashboards hold potential to help individuals self-regulate their behaviors and compare themselves with their peers' academic success [24].

While these dashboards may support intervention strategies, as well as facilitate personalized education, many scholars note that they enable faculty to profile students based on their risk status and create conditions where students experience the phenomena of self-fulfilling prophecies. Citing Greller and Drachsler [25], Roberts et al. [26, p. 2] argue that learning analytics may contribute to poor faculty decision making, where they judge students "based on a limited set of parameters [, creating] a context for profiling, and profiling can result in limiting students' potential and damaging self-efficacy." Rubel and Jones [16] raise another point regarding fairness. It may be so that faculty, who know which students are predicted to succeed and those who are not, will turn their focus and labor toward students who will 1) not be a burden on their time and 2) will add value to the course's learning community. Clearly, the distribution of resources in this hypothetical case is unfair-but plausible. More recently, the learning analytics community has seriously considered whether or not fairness and bias issues are becoming embedded in the predictive measures and machine learning algorithms that drive them [27], [28].

An emerging set of the learning analytics literature concerns issues related to faculty, for the surveillant eye of learning analytics easily turns to faculty who are themselves embedded in and reliant upon institutional information infrastructures like their students. The datafication of instruction and student learning is further enabling faculty monitoring and scoring in ways that parallel student-focused learning analytics. Williamson et al. [29, pp. 357-358] remind us that "datafication also demands certain kinds of classroom pedagogies. Not all forms of learning can be quantified and analyzed. And this means, potentially, that not all forms of teaching and learning will 'count' in terms of how teachers and students are measured and assessed." While faculty (so far) retain academic freedom rights to make instructional choices 
they see fit to their students' educational needs and disciplinary norms, increasing surveillance and decreasing faculty power potentially make these institutional actors subject to invasive management and autonomy-limiting digital governance [30]. Leading learning analytics researchers argue that the methods and measures they advocate for should not be used against faculty [see 33]; however, administrative "techno-solutionism" [32] continues unabated in higher education, especially where audit culture and neoliberalism motivate leadership decisions and the restructuring of the academy [33]-[35].

\subsection{Faculty perspectives and problems}

Research on user perspectives of learning analytics, much like the ethics of the sociotechnical practice, remains focused on students. For example, some research raises questions about privacy rights and system design expectations ([20], [26], see [36][38]). But what of faculty? It is surprising that faculty perspectives of learning analytics are not a widely studied topic, save a few key articles [39]-[41].

Where research has been done, it has focused on faculty adoption, specifically how to incentive tool use and make data-based decisions [39], [40], [42], [43]. Some of the research has uncovered emerging privacy concerns. A reoccurring issue present in a number of studies is the perception that learning analytics presents a "big brother" type environment [41]-[46]. However, this literature set did not fully develop what faculty mean when they claim that analytics were "big brother-ish." Outside of general privacy concerns, we noted two primary ethics issues. The first concerns student profiling. Faculty argued that existing data is insufficient to allow for a holistic student view [41], [44], [45], which could lead to biased or incorrect actions against students [42], [43], [45]. The second issue is more personal to faculty and matches the points raised by ethicists. Parrish and Richman [46, p. 10] found that faculty were keenly aware of the possibility for academic freedom to be lessened, writing that they "expect the opportunity to make their own legitimate judgments about the content, their teaching, and what their students need in order to be successful in higher education coursework."

\subsection{Emerging research questions}

Faculty are arguably on the front line of student privacy. Their tool choices, instructional designs, and course policies impact the degree to which students retain privacy. That there is little known about how faculty interpret the privacy issues and then take action to limit them signifies a noteworthy gap in the literature worthy of inquiry.

This paper marks the first effort of a multi-phase, grant-funded research agenda to better understand this underdeveloped area. We argue that it is necessary to begin research in this space by understanding faculty views of privacy, both personally and with regard to students. Definitions of, perspectives in relation to, and the value of privacy can and does differ [47], [48]. We need to begin this work by first understanding faculty views on student privacy before moving into an actionable space (e.g., policy recommendations, technological design choices, instructional approaches).

Using a survey methodology that we describe below, our exploratory research questions are:

- RQ 1: Do faculty value their privacy?

- RQ 2: Do faculty value their students' privacy?

Future research from this survey will address, among other things, faculty use of learning analytics, student privacy training, knowledge of student privacy resources, and how student privacy influences instructional decision making.

\section{Methods}

\subsection{Survey design and evaluation}

We designed the study using a multi-step strategy. First, we drafted the initial survey based on an extensive literature review. Next, we made edits to its structure, removing overlapping, redundant and tangential questions. We then thematically organized questions into seven sections covering privacy demographics, privacy perceptions and expectations, privacy practices, privacy policy, learning analytics, personal demographics, and professional demographics. Following this step, the project's expert advisory board provided its input, which led to further culling and question refining. Finally, we conducted ten cognitive interviews with participants who fit the sample's constraints using a draft of the survey; we made changes based on the results of the cognitive interviews.

\subsection{Survey distribution procedures}

The final survey consisted of 62 questions, which we fielded using the Qualtrics Panel Systems. Qualtrics recruited participants who self-identified as full-time instructors in the United States from nonprofit colleges and universities. An initial 72-hour 
launch yielded 40 responses; we used these data to test survey functionality. Following a review, we fully distributed the survey. To achieve a confidence level of $95 \%$ with a $+/-5$ confidence interval for reporting results, we determined that a sample size of at least 385 participants would be needed from the population of approximately 1.5 million higher education faculty in the United States [49]. As chi-square analysis was planned for data analysis, this sample size was also needed to achieve the required test power $(\beta=.80)$ in order to identify at least a medium effect size (0.3). In total, Qualtrics fielded responses for 42 days in early 2020, yielding a final total of 502 viable responses for data analysis.

\subsection{Personal and professional demographics}

While we did not intend to achieve a nationally representative sample of higher education faculty, our demographics are arguably representative across a number of categories - and usefully oversampled in some instances - tracked by the National Center for Education Statistics [49], [50]. The most recent national numbers indicate that faculty are split across traditional gender lines (i.e., male and female), and our survey sampled slightly more female-identifying $(56.8 \%)$ than male-identifying faculty. Our ethnicity/race demographics are comparable as well; see Table 1.

\section{Table 1. Race and Ethnicity Response Rates as Compared with National Rates}

\begin{tabular}{lll} 
Race/Ethnicity & Response Rate & National Rate \\
\hline White & $78.3 \%$ & $68.8 \%$ \\
\hline Black & $5.8 \%$ & $5.5 \%$ \\
\hline Hispanic & $6 \%$ & $4.9 \%$ \\
\hline $\begin{array}{l}\text { Asian/Pacific } \\
\text { Islander }\end{array}$ & $10.3 \%$ & $9.6 \%$ \\
\hline $\begin{array}{l}\text { Native } \\
\text { American }\end{array}$ & $1.6 \%$ & $0.4 \%$ \\
\hline
\end{tabular}

Finally, our respondents reflect similar percentages regarding institutional representation. For public versus private institutional percentages, the survey ratio is $69.1 \% / 30.9 \%$ and the national ratio is $63.6 \% / 36.4 \%$.

Age range distribution was fairly balanced, with slightly more falling in the 35 to 44 range (32.1\%), followed by 22 to $34(24.1 \%), 45$ to $54(22.3 \%)$, and 55 to $64(15.7 \%)$. Only $4.8 \%$ of respondents identified as being 65 and over, with five opting to not respond. Sexual orientation indicated that faculty were primarily straight $(83.7 \%)$.
Politically, those who identified as Democratic made up nearly half of the respondents, though a large group $(26.1 \%)$ identified as having "no political affiliation or independent." Nearly half identified as being Christian (47.6\%), followed by "no religious affiliation" (14.5\%), "agnostic" (11.4\%), "Atheist" $(10.4 \%)$, with the remainder representing a wide variety of religious affiliations. Most respondents did not identify as having a disability/ability status, and most also did not identify as being an immigrant.

Respondents were asked about their professional demographics. Most participants identified as full professors $(23.5 \%)$, though other titles were well represented with $20.9 \%$ assistant professors, $17.9 \%$ associate professors, $16.5 \%$ adjuncts, and $15.1 \%$ lecturers; $5.4 \%$ indicated a title not listed and only three respondents $(0.6 \%)$ identified as professor emeritus. Response rates by field are in Table 2 .

\section{Table 2. Field Representation Response Rates}

\begin{tabular}{ll} 
Field & Response Rate \\
\hline $\begin{array}{l}\text { Science, Technology, } \\
\text { Engineering, \& Medicine } \\
\text { (STEM) }\end{array}$ & $28.9 \%$ \\
\hline Arts and Humanities & $24.1 \%$ \\
\hline Social and Behavioral Sciences & $18.1 \%$ \\
\hline Education & $12.2 \%$ \\
\hline Business & $7.2 \%$ \\
\hline A Field Not Listed & $5.4 \%$ \\
\hline Performing Arts & $4.2 \%$ \\
\hline
\end{tabular}

On a sliding scale, respondents were asked if they identify more as a teacher or a researcher. $25.1 \%$ of respondents identified as $100 \%$ teacher, while only 3 $(0.6 \%)$ respondents identified as $100 \%$ researcher. Most fell in between both identities, though more $(59.0 \%)$ selected a range that was more teacher than researcher.

There was a good balance in terms of years of instructional experience and format of instruction. $21.7 \%$ of respondents selected 0 to 5 years of experience, $22.9 \%$ selecting 6 to 10 years, $21.3 \%$ selecting 11 to 15 years, $13.7 \%$ selecting 16 to 20 years, and $20.3 \%$ selecting 21 years or more. Face-toface $(63.1 \%)$ was the most often selected format in which respondents primarily teach, followed by hybrid $(29.9 \%)$ and online $(7.0 \%)$.

Student populations primarily taught skewed toward undergraduates $(69.1 \%)$, followed by a combination of undergraduates and graduates $(21.3 \%)$, and solely graduates $(9.6 \%)$. Class size was balanced between those teaching 21-50 students 
(48.4\%) and those teaching 0-20 students (41.8\%); very few respondents taught more than 50 students.

\section{Findings}

\subsection{Defining and valuing privacy}

We asked participants to rank six of the more common definitions of privacy (see [47], [48]), putting the definition that most resonates with their understanding of privacy in the first of six positions. The definitions were as follows, which we have prefixed with a shorthand:

- Contextual Privacy: Privacy determines the flow of information (e.g., access, re-use, disclosure) about me in a given context (e.g., healthcare, education)

- Relational Privacy: Privacy enables me to form relationships involving respect, love, friendship, and trust.

- Information Control: Privacy lets me control the information that is generated about me.

- Information Access: Privacy lets me limit other people's access to information about me.

- Intellectual Privacy: Privacy protects me when I'm doing intellectual activities (e.g., searching for information, writing, thinking).

- Personal Privacy: Privacy protects my dignity, autonomy, and independence.

$33.8 \%(\mathrm{n}=170)$ of participants indicated that the information access definition most resonated with their understanding of privacy; the personal privacy definition followed with $22.1 \%(\mathrm{n}=111)$. When located in the second ranking position, information access still remained at the top $(31.1 \%, \mathrm{n}=156)$, but it was followed by the control definition $(22.5 \%, \mathrm{n}=$ 113). $48.8 \%(n=245)$ ranked relational privacy in the sixth position, indicating that it resonated the least with their definition of privacy.

Asking participants to reveal how they define privacy does not disclose whether or not they value it. So, we followed our definitional question with a Likert scale question with this stem: How important to you is your personal privacy? $63.3 \%(n=318)$ stated that privacy was very important to them, $32.9 \%(\mathrm{n}=165)$ selected that it was important, and $4 \%(n=20)$ indicated that it was moderately important. No participants stated that privacy was only slightly important or not important.

\subsection{Identity effects on privacy}

Research has shown that perspectives on privacy are often influenced by demography and identity. This is due in part to some demographics, such as one's race or religion, being less socioeconomically privileged. Protecting information disclosures may be a necessary defense or one seen as an advantageous move. We asked participants if any of their demographic or identity markers influenced their privacy views. 50\% $(\mathrm{n}=251)$ stated that their political affiliation was influential, followed closely by their age $(45.8 \%, \mathrm{n}=$ $230)$, and their religious affiliation $(41.8 \%, \mathrm{n}=210)$. Participants were less likely to state that immigration status $(15.1 \%, \mathrm{n}=76)$, disability/ability status $(23.3 \%$, $\mathrm{n}=117)$, or ethnic identity $(26.9 \%, \mathrm{n}=135)$ influenced their privacy views. Additional chi-square analyses identified significant results.

There proved to be significant findings between respondents identifying as immigrants and their views on privacy, yielding a significant association $\left(\chi^{2}=\right.$ $26.03, \mathrm{df}=2, \mathrm{p}<0.0012)$ with a medium effect size (Cramer's $\mathrm{V}=0.27$ ). And there was a significant association $\left(\chi^{2}=18.13, \mathrm{df}=5, \mathrm{p}<0.0029\right)$ with a medium effect size (Cramer's $\mathrm{V}=0.19$ ) between whether immigration status influences their view of privacy and the rating of relational privacy. A Bonferroni post hoc analysis indicated that those who indicated that their immigration status influenced their view on privacy more often than expected ranked the relational privacy second or third in terms of how well the definition resonated with their understanding of privacy. There was a significant association $\left(\chi^{2}=\right.$ $19.10, d f=2, \mathrm{p}<0.0012$ ) with a medium effect size (Cramer's $\mathrm{V}=0.23$ ) between immigration status and whether ethnic identity influenced their view of privacy. A Bonferroni post hoc analysis indicated that those who identified as an immigrant more often than expected indicated that their ethnic identity influenced their views on privacy.

Respondents' political affiliations also bore interesting findings. For instance, one's political affiliation is intertwined with their religious affiliation, which influences views on privacy. Chi-square analysis uncovered a statistically significant difference $(\chi 2=19.58, \mathrm{df}=4, \mathrm{p}<0.0012)$, with a medium effect size (Cramer's $\mathrm{V}=0.20)$. A Bonferroni post hoc analysis indicated that those who affiliated as Republican more often than expected indicated that their religious affiliation influenced their views on privacy compared to those who affiliated as Democratic or as having no political affiliation. And those who identified as having no political affiliation more often than expected indicated that their religious affiliation did not influence their views on privacy compared to those who affiliated as Republicans or Green, Libertarian, \& Other. 
The findings show how some aspects of demography influence faculty views of their privacy, but it is striking that there is very little other statistical difference to report. No statistically significant associations were found between aspects of respondents' professional identity and how faculty view their personal privacy or student privacy. We expected that, at the least, whether one view one's self more as a teacher or researcher would demonstrate a statistical effect, but it did not. Our driving assumption was that faculty whose professional identity is tied more to teaching and student support than research would be correlated with a higher value related to student privacy.

\subsection{Defining and valuing student privacy}

How a faculty member defines and ranks their own view of privacy may change when considering students. To home in on this question, we duplicated the question we addressed in section 4.1 with minor changes, namely modifying the pronouns to instead refer to students. As with participants' own views of privacy, the data indicate that faculty perceive information access as the definition of privacy most applicable to students $(32.9 \%, \mathrm{n}=165$; this reflects a downward change of 1\%). Again, as with participants' views of their privacy, faculty chose relational privacy as the definition that least reflected their perception of student privacy. Across all six placements, there are no notable percentage or ranking changes.

We sought to identify if faculty valued their students' privacy: the result was overwhelming. Using a Likert scale, we determined that $98.9 \%$ of respondents stated that privacy was either very important $(74.8 \%, \mathrm{n}=376)$ or important $(24.1 \%, \mathrm{n}=$ 121). No faculty indicated that their students' privacy was not important. Given privacy's instrumental role in the construction and protection of intellectual artifacts and behaviors often associated with learning, we asked if student privacy was important with regard to the following:

- building relationships with their student peers,

- building course learning communities,

- achieving course learning outcomes,

- developing ideas,

- sharing ideas,

- and creating course artifacts (e.g., papers, presentations, online discussion posts).

Across all measures, a majority of faculty stated that privacy was either very important or important.
Relationship building was the highest scored measure with an aggregate percentage of $74 \%(n=372)$.

Even if faculty believe that student privacy is important and see value in it as part of the learning process, it does not necessarily follow that they perceive that students care about their privacy. $80.1 \%$ $(\mathrm{n}=402)$ stated that they believed students care about their privacy, $8.4 \%(\mathrm{n}=42)$ stated that students did not care, and $11.6 \%(\mathrm{n}=58)$ were unsure if students cared.

Additional analysis using Spearman's rho found positive, but weak, correlations between how important respondents felt student privacy was and the importance of privacy for students' ability to perform course related activities. The more important respondents felt student privacy was, the more likely they were to feel that privacy was important or very important for students' ability to build relationships with their student peers $\left(r_{s}=0.19, p<.0025\right)$, build course learning communities $\left(r_{s}=0.20, p<.0025\right)$, achieve course learning outcomes $\left(r_{s}=0.18, p\right.$ $<.0025)$, develop ideas $\left(r_{s}=0.14, p<.0025\right)$, share ideas $\left(r_{s}=0.17, p<.0025\right)$, and creating course artifacts (e.g., papers, presentations, online discussion posts) $\left(r_{s}=0.15, p<.0025\right)$.

\section{Discussion}

\subsection{Values and perspectives}

Important findings from this research suggest that which was previously unknown, especially given the new sociotechnical context of learning analytics: faculty care deeply about their privacy and their students' privacy. Moreover, they define their own privacy and their students' privacy through the same conceptual lens of information access. Finally, they agree with their peers that students care about their privacy.

We are also left to consider the parallel conceptual approach to faculty's personal privacy and student privacy. We questioned whether or not faculty would rank intellectual privacy highly for students given privacy's instrumental role in the pursuit of ideas and development of speech. This idea was proven wrong. In the first placement of definitions, intellectual privacy ranked second-to-last. Faculty identify privacy as important for learning, so the conceptual misalignment is notable.

Given the importance of student privacy to faculty, it should follow that faculty proactively develop privacy protections and are vocal supporters of student privacy. But a study of faculty syllabi indicated that student privacy was a rarely addressed topic in such a key instructional document [51]. The authors postulate that the finding "means that 
instructors find [privacy] to be neither instrumental to learning nor intrinsically valuable. Thus, instructors believe it is simply not necessary to discuss privacy in their syllabi” [51, p. 1347]. Our findings run counter to this claim.

\subsection{Continuing questions}

The findings raise questions about privacy values in the context of learning analytics. In particular, the findings indicate that faculty uniformly value student privacy. However, the classroom is a complicated environment of competing values and these findings do not reveal how student privacy compares with other values that faculty find important.

The learning analytics literature puts privacy in conflict with other values, such as institutional efficiency and student learning [52], but research is needed to understand the relative value of privacy from the faculty perspective to compare faculty values with those of other stakeholders, such as administrators, students, and instructional designers. In combination with other literature suggesting that students do care about their privacy, the findings herein indicate that faculty care about students' privacy as well. Actors with power who claim that student privacy is not valued do so wrongly. This research begins to balance the power dynamic, giving students and faculty empirical findings to use as leverage to change policy, practice, and technological design.

It is unclear whether the perspective on student privacy revealed by this study, namely its importance and its focus on information access, comes from an informed and reflective position. Some instructors indicated at the end of the survey that student privacy was not something they had spent extensive time thinking about, for example: "This gave me some things to think about!" and "This was a very interesting survey, primarily b/c I'd never thought about privacy in the manner or with the breadth with which it is dealt in this survey." For others the experience of taking the survey exposed their lack of knowledge about privacy issues: "it showed me how much i do NOT know about privacy laws and policies." Further, research demonstrates that faculty's instructional practices do not always reflect their espoused beliefs [53], [54].

Research is needed to explore the effect of faculty' understanding and value of student privacy on their classroom practices. More analysis of the survey data will help with this gap. In addition, further phases of this grant-funded project, including interviews with faculty and focus groups with a variety of campus stakeholders, will provide deeper insights.

\section{Conclusion}

Learning analytics raises the specter of student privacy invasions in ways once unimaginable. While this sociotechnical movement continues to gain momentum, higher education institutions are attempting to calibrate their ethical compasses, develop appropriate policies, and adjust practices. Instructors are at the front line of student privacy, and their values and perspectives will affect how and if students retain privacy in physical and virtual classrooms. Our research begins to provide light on this subject by highlighting that, indeed, faculty find student privacy to be important and see its value in the classroom. Our future research will unpack how these views play out in instructional situations and when learning analytics are utilized.

\section{References}

[1] D. Gašević, S. Dawson, and G. Siemens, "Let's not forget: Learning analytics are about learning," TechTrends, vol. 59, no. 1, pp. 64-71, Jan. 2015, doi: 10.1007/s11528-014-0822-x.

[2] G. Siemens, "Learning analytics: The emergence of a discipline," American Behavioral Scientist, vol. 57, no. 10 , pp. $1380-1400$, Oct. 2013 , doi: $10.1177 / 0002764213498851$.

[3] B. Williamson, "Digital education governance: Data visualization, predictive analytics, and 'real-time' policy instruments," Journal of Education Policy, vol. 31, no. 2, pp. 123-141, Mar. 2016, doi: 10.1080/02680939.2015.1035758.

[4] B. Williamson, "Policy networks, performance metrics and platform markets: Charting the expanding data infrastructure of higher education," British Journal of Educational Technology, vol. 50, no. 6, pp. 27942809, 2019, doi: 10.1111/bjet.12849.

[5] S. Goldrick-Rab, Paying the Price: College Costs, Financial Aid, and the Betrayal of the American Dream. Chicago: University of Chicago Press, 2016.

[6] K. M. L. Jones, A. Rubel, and E. LeClere, "A matter of trust: Higher education institutions as information fiduciaries in an age of educational data mining and learning analytics," Journal of the Association for Information Science and Technology, to be published, doi: 10.1002/asi.24327.

[7] R. Clarke, "Big data, big risks," Information Systems Journal, vol. 26, no. 1, pp. 77-90, 2016, doi: 10.1111/isj.12088.

[8] L. Floridi, "Big data and their epistemological challenge," Philosophy \& Technology, vol. 25, no. 4, pp. 435-437, Dec. 2012, doi: 10.1007/s13347-0120093-4.

[9] D. Beer, The Data Gaze: Capitalism, Power and Perception. Thousand Oaks, CA: SAGE Publications, 2018. 
[10] D. J. Solove, The Digital Person: Technology and Privacy in the Information Age. New York, NY: NYU Press, 2004.

[11] K. M. L. Jones, "Learning analytics and higher education: A proposed model for establishing informed consent mechanisms to promote student privacy and autonomy," International Journal of Educational Technology in Higher Education, vol. 16, no. 1, Jul. 2019, doi: 10.1186/s41239-019-0155-0.

[12] M. Kwet and P. Prinsloo, "The 'smart' classroom: A new frontier in the age of the smart university," Teaching in Higher Education, vol. 25, no. 4, pp. 510 526, May 2020, doi: $10.1080 / 13562517.2020 .1734922$.

[13] J. Fritz and J. Whitmer, "Ethical learning analytics: 'Do no harm' versus 'do nothing,", New Directions for Institutional Research, vol. 2019, no. 183, pp. 2738, 2019, doi: 10.1002/ir.20310.

[14] R. Ferguson, "Ethical challenges for learning analytics," Journal of Learning Analytics, vol. 6, no. 3, pp. 25-30-25-30, Dec. 2019, doi: 10.18608/jla.2019.63.5.

[15] A. Pardo and G. Siemens, "Ethical and privacy principles for learning analytics," British Journal of Educational Technology, vol. 45, no. 3, pp. 438-450, 2014, doi: 10.1111/bjet.12152.

[16] A. Rubel and K. M. L. Jones, "Student privacy in learning analytics: An information ethics perspective," The Information Society, vol. 32, no. 2, pp. 143-159, Mar. 2016, doi: 10.1080/01972243.2016.1130502.

[17] V. Scholes, "The ethics of using learning analytics to categorize students on risk," Educational Technology Research and Development, vol. 64, no. 5, pp. 939955, Oct. 2016, doi: 10.1007/s11423-016-9458-1.

[18] N. Selwyn, "Re-imagining 'learning analytics' ... a case for starting again?," The Internet and Higher Education, vol. 46, Jul. 2020, doi: 10.1016/j.iheduc.2020.100745.

[19] S. Slade and P. Prinsloo, "Learning analytics: Ethical issues and dilemmas," American Behavioral Scientist, vol. 57, no. 10, pp. 1510-1529, Oct. 2013, doi: $10.1177 / 0002764213479366$.

[20] K. M. L. Jones et al., "'We're being tracked at all times': Student perspectives of their privacy in relation to learning analytics in higher education," Journal of the Association for Information Science and Technology, to be published, doi: 10.1002/asi.24358.

[21] S. Hartman-Caverly, "Human nature is not a machine: On liberty, attention engineering, and learning analytics," Library Trends, vol. 68, no. 1, pp. 24-53, Oct. 2019, doi: 10.1353/lib.2019.0029.

[22] J. Polonetsky and O. Tene, "Who is reading whom now: Privacy in education from books to MOOCs," Vanderbilt Journal of Entertainment \& Technology Law, Oct. 2014, [Online]. Available: http://heinonlinebackup.com/hol-cgibin/get_pdf.cgi?handle=hein.journals/vanep17\&sectio $\mathrm{n}=31$.

[23] E. Duval, "Attention please! Learning analytics for visualization and recommendation," in Proceedings of the 1st International Conference on Learning Analytics and Knowledge, Banff, Alberta, Canada, Feb. 2011, pp. 9-17, doi: 10.1145/2090116.2090118.

[24] R. Bodily and K. Verbert, "Review of research on student-facing learning analytics dashboards and educational recommender systems," IEEE Trans. Learn. Technol., vol. 10, no. 4, pp. 405-418, Oct. 2017, doi: 10.1109/TLT.2017.2740172.

[25] W. Greller and H. Drachsler, "Translating learning into numbers: A generic framework for learning analytics," Journal of Educational Technology \& Society, vol. 15, no. 3, pp. 42-57, 2012, [Online]. Available: https://www.jstor.org/stable/jeductechsoci.15.3.42.

[26] L. D. Roberts, J. A. Howell, K. Seaman, and D. C. Gibson, "Student attitudes toward learning analytics in higher education: 'The Fitbit version of the learning world," Frontiers in Psychology, vol. 7, 2016, doi: 10.3389/fpsyg.2016.01959.

[27] K. Holstein and S. Doroudi, "Fairness and equity in learning analytics systems (FairLAK)," in Companion Proceedings of the Ninth International Learning Analytics \& Knowledge Conference (LAK 2019), Tempe, AZ, USA, 2019, pp. 1-2.

[28] R. Marachi and L. Quill, "The case of Canvas: Longitudinal datafication through learning management systems," Teaching in Higher Education, vol. 25, no. 4, pp. 418-434, May 2020, doi: 10.1080/13562517.2020.1739641.

[29] B. Williamson, S. Bayne, and S. Shay, "The datafication of teaching in Higher Education: Critical issues and perspectives," Teaching in Higher Education, vol. 25, no. 4, pp. 351-365, May 2020, doi: 10.1080/13562517.2020.1748811.

[30] L. Castañeda and N. Selwyn, "More than tools? Making sense of the ongoing digitizations of higher education," International Journal of Educational Technology in Higher Education, vol. 15, no. 1, p. 22, May 2018, doi: 10.1186/s41239-018-0109-y.

[31] N. Selwyn and D. Gašević, "The datafication of higher education: discussing the promises and problems," Teaching in Higher Education, vol. 25, no. 4, pp. 527540, May 2020, doi: 10.1080/13562517.2019.1689388.

[32] E. Morozov, To Save Everything, Click Here: The Folly of Technological Solutionism. New York, NY: PublicAffairs, 2013.

[33] R. Hall, "Technology-enhanced learning and cooperative practice against the neoliberal university," Interactive Learning Environments, vol. 24, no. 5, pp. 1004-1015, Jul. 2016, doi: 10.1080/10494820.2015.1128214.

[34] P. Prinsloo and S. Slade, "Retracing the evolution of thinking ethically about student data," New Directions for Institutional Research, vol. 2019, no. 182, pp. 1934, 2019, doi: 10.1002/ir.20305.

[35] N. Selwyn, "Data entry: Towards the critical study of digital data and education," Learning, Media and Technology, vol. 40, no. 1, pp. 64-82, Jan. 2015, doi: 10.1080/17439884.2014.921628. 
[36] L. Bennett and S. Folley, "Four design principles for learner dashboards that support student agency and empowerment," Journal of Applied Research in Higher Education, vol. 12, no. 1, pp. 15-26, Jan. 2019, doi: 10.1108/JARHE-11-2018-0251.

[37] D. Ifenthaler and C. Schumacher, "Student perceptions of privacy principles for learning analytics," Educational Technology Research and Development, vol. 64, no. 5, pp. 923-938, Oct. 2016, doi: 10.1007/s11423-016-9477-y.

[38] C. Schumacher and D. Ifenthaler, "Features students really expect from learning analytics," Computers in Human Behavior, vol. 78, no. January 2018, pp. 397407, Jan. 2018, doi: 10.1016/j.chb.2017.06.030.

[39] N. Arthars and D. Liu, "How and why faculty adopt learning analytics: Wide-scale learning analytics adoption through a 'diffusion of innovation' lens," preprint, p. 17.

[40] M. T. Hora, J. Bouwma-Gearhart, and H. J. Park, "Data driven decision-making in the era of accountability: Fostering faculty data cultures for learning," The Review of Higher Education, vol. 40, no. 3, pp. 391-426, Mar. 2017, doi: 10.1353/rhe.2017.0013

[41] C. Klein, J. Lester, H. Rangwala, and A. Johri, "Learning analytics tools in higher education: Adoption at the intersection of institutional commitment and individual action," The Review of Higher Education, vol. 42, no. 2, pp. 565-593, 2019, doi: 10.1353/rhe.2019.0007.

[42] B. Dietz-Uhler and J. E. Hurn, "Using learning analytics to predict (and improve) student success: A faculty perspective," Journal of Interactive Online Learning, vol. 12, no. 1, pp. 17-26, 2013.

[43] D. Bollenback and A. Glassman, "Big data in higher education: Adjunct faculty perceptions of learning analytics and their uses," Issues in Information Systems, vol. 19, no. 3, pp. 71-80, 2018.

[44] O. Khan, 'Learners' and Teachers' Perceptions of Learning Analytics (LA): A Case Study of Southampton Solent University (SSU)," presented at the International Conference on Cognition and Exploratory Learning in Digital Age, Vilamoura, Algarve, Portugal, Oct. 2017, Accessed: Jun. 09, 2020. [Online]. Available: https://eric.ed.gov/?id=ED579477.

[45] D. B. Knight, C. Brozina, and B. Novoselich, “An investigation of first-year engineering student and instructor perspectives of learning analytics approaches," Journal of Learning Analytics, vol. 3, no. 3, pp. 215-238, 2016, doi: 10.18608/jla.2016.33.11.

[46] A. H. Parrish and L. J. Richman, "Dual perspectives on learning analytics in higher education," Journal of Applied Research in Higher Education, vol. 12, no. 1, pp. 4-14, Jan. 2019, doi: 10.1108/JARHE-12-20180254.

[47] H. Nissenbaum, Privacy in Context: Technology, Policy, and the Integrity of Social Life. Stanford University Press, 2009.
[48] D. J. Solove, “A taxonomy of privacy," University of Pennsylvania Law Review, vol. 154, no. 3, pp. 477560, 2005.

[49] National Center for Education Statistics, "Number of faculty in degree-granting postsecondary institutions, by employment status, sex, control, and level of institution: Selected years, fall 1970 through fall 2018," 2019.

https://nces.ed.gov/programs/digest/d19/tables/dt19_3 15.10.asp (accessed Jul. 13, 2020).

[50] National Center for Education Statistics, "Full-time faculty in degree-granting postsecondary institutions, by race/ethnicity, sex, and academic rank: Fall 2015, fall 2017, and fall 2018," 2019. https://nces.ed.gov/programs/digest/d19/tables/dt19_3 15.20.asp (accessed Jul. 13, 2020).

[51] K. M. L. Jones and A. VanScoy, "The syllabus as a student privacy document in an age of learning analytics," Journal of Documentation, vol. 75 , no. 6 , pp. 1333-1355, Jan. 2019, doi: 10.1108/JD-12-20180202.

[52] T. Oliphant and M. R. Brundin, "Conflicting values: An exploration of the tensions between learning analytics and academic librarianship," Library Trends, vol. 68 , no. 1 , pp. 5-23, Oct. 2019, doi:

10.1353/lib.2019.0028.

[53] M. M. Buehl and J. S. Beck, "The relationship between teachers' beliefs and teachers' practices," in International Handbook of Research on Teachers' Beliefs, New York, NY: Routledge, 2015, pp. 66-84.

[54] R. Kane, S. Sandretto, and C. Heath, "Telling half the story: A critical review of research on the teaching beliefs and practices of university academics," Review of Educational Research, vol. 72, no. 2, pp. 177-228, Jun. 2002, doi: 10.3102/00346543072002177.

\section{Acknowledgements}

This project was made possible in part by the Institute of Museum and Library Services (LG-1819-0032-19). The views, findings, conclusions, or recommendations expressed in this publication do not necessarily represent those of the Institute of Museum and Library Services. We thank our participants for taking time to complete the survey. Some survey questions were adopted and modified from the Data Doubles (https://datadoubles.org) student privacy survey. We thank the research team members for allowing us to iterate on their work. 